\title{
O CUIDADO E A APRENDIZAGEM DAS PESSOAS COM SÍNDROME DE DOWN: REVISÃO DE LITERATURA
}

\section{Eduarda Rodrigues Machado'; Flávia Dorneles Saleh2; João Victor Quartieri Simões Pires; ; Munah Najeh Saleh Ahmad Maruf ${ }^{4}$; Ana Luiza Rios Antunes; Luiza Fortes Lamberty ${ }^{6}$; Bianca Baldin; Maria Helena Gehlen ${ }^{8}$}

\section{RESUMO}

Objetivo: Socializar a construção do portfólio acadêmico no desenvolvimento da temática que atende as necessidades do cuidado com as pessoas com Síndrome de Down. Metodologia: Trata-se de um relato de experiência desenvolvido como atividade de ensino no terceiro semestre no curso de Enfermagem na Universidade Franciscana. O período foi no primeiro semestre de 2021. Nos resultados, evidenciou-se a necessidade das competências, habilidades e atitudes do enfermeiro, bem como na formação profissional de elencar pressupostos para o atendimento das necessidades de cuidados e educação inclusiva no desenvolvimento da pessoa com Síndrome de Down. Conclusão: Ao socializar a construção do portfólio acadêmico no desenvolvimento da temática que atende as necessidades do cuidado com as pessoas com Síndrome de Down, entende-se a sua relevância no sentido de possibilitar ao estudante o efetivo sentido de refletir acerca das temáticas que emergem da formação profissional e que necessitam ser discutidas com protagonismo e autonomia.

\footnotetext{
${ }^{1}$ Acadêmica do Curso de Enfermagem da Universidade Franciscana - UFN. eduardamachado886@gmail.com ${ }^{2}$ Acadêmica do Curso de Enfermagem da Universidade Franciscana - UFN. flaviasaleh222@gmail.com ${ }^{3}$ Acadêmico do Curso de Enfermagem da Universidade Franciscana - UFN. quarti.joao@gmail.com ${ }^{4}$ Acadêmica do Curso de Enfermagem da Universidade Franciscana - UFN. Munahsaleh1999@hmail.com ${ }^{5}$ Acadêmica do Curso de Enfermagem da Universidade Franciscana - UFN. riosana1306@gmail.com ${ }^{6}$ Acadêmica do Curso de Enfermagem da Universidade Franciscana - UFN. luizaflamberty@gmail.com 7Enfermeira pela Universidade Franciscana - UFN. biancavbaldin@gmail.com 8Enfermeira. Doutora em Enfermagem. Professora do curso de Enfermagem da UFN. gehlenmh@gmail.com
} 
Palavras- chave: Síndrome de Down, Família, Enfermagem

Eixo Temático: Atenção Integral e Promoção à Saúde (AIPS)

\section{INTRODUÇÃO}

A Síndrome de Down é uma alteração genética no cromossomo 21 , que é causada quando a divisão celular acontece de forma anormal, resultando assim em um material extra no cromossomo 21. Essa condição genética pode aparecer de 3 formas, nas quais são: Trissomia 21, Mosaicismo e Translocação (LEITE et al 2018).

Entre as características mais frequentes pode-se expor: hipotonia muscular, língua protusa, olhos amendoados, mãos com linha única, cardiopatias congênitas e atraso no desenvolvimento motor e intelectual. Além das características já citadas, as crianças têm uma personalidade muito afetuosa, que esbanja amor e carinho com as pessoas em sua volta (SANTANA; CAVALCANTE, 2018).

Segundo Hannum; et al (2021), a chegada de uma criança com Síndrome de Down na família é um momento onde muitos sentimentos, pensamentos, dúvidas, medos e incertezas prevalecem, pois é algo desconhecido, no qual não irá afetar apenas o presente, mas sim o futuro da criança e da família. Dessa forma, a sobrecarga, tanto financeira e física quanto social e psicológica é visível, pois a preocupação com o desenvolvimento do infante é maior. Por esse motivo, a família busca ajuda de profissionais e terapias qualificadas para ajudar no crescimento e desenvolvimento dessa criança. (NUNES; DUPAS, 2011).

A família é o primeiro contato da sociedade que a criança vai receber, sendo os protagonistas na evolução, desenvolvimento e aprendizado, tanto em níveis físicos e intelectuais quanto em níveis sociais. Apesar das dificuldades, é imprescindível que a família crie estratégias para potencializar o desenvolvimento do seu filho, pois a cada conquista, a família se motiva mais (MATIAS DE LA CRUZ; SALVADOR; VELENZUELA, 2021).

O profissional Enfermeiro, tem como principal objetivo de atuação um caráter interdisciplinar, para promover, proteger e recuperar a saúde, desse modo, fornecer 
um atendimento de qualidade aos pacientes. Nesse sentido, a promoção dos cuidados com uma pessoa com necessidade especial de aprendizagem é muito importante desde o começo da gestação, pois permite fornecer orientações às famílias sobre os cuidados e necessidades especiais dessa criança (ALVES; PIRES; SERVO, 2013).

Com base no exposto, objetiva-se assim, socializar a construção do portfólio acadêmico no desenvolvimento da temática que atende as necessidades do cuidado com as pessoas com Síndrome de Down.

\section{METODOLOGIA}

Trata-se de um relato de experiência desenvolvido como atividade de ensino no terceiro semestre no curso de graduação de Enfermagem na Universidade Franciscana- UFN, no qual foi construído o portfólio acadêmico como boa prática pedagógica, que entregou as competências e habilidades da formação profissional, aliando o interesse pessoal para o desenvolvimento da temática Síndrome de Down e a sua relevância nas atitudes profissionais da promoção do cuidado da enfermagem. O período foi no primeiro semestre de 2021. De tal modo, foi construído com base nas seguintes etapas: introdução, desenvolvimento, com entrevistas aos familiares, profissionais de saúde com articulação das evidências por meio de uma revisão de literatura.

Foram considerados os artigos publicados na biblioteca virtual Scielo, no banco de dados eletrônicos da Literatura Latino-Americana e do Caribe em Ciências da Saúde (LILACS). Os descritores foram estabelecidos e classificados em Ciências da Saúde (DeCS). O projeto não passou pelo comitê de ética por se tratar de um relato de experiência.

\section{RESULTADOS E DISCUSSÕES}

Foi possível perceber que a Síndrome de Down é uma alteração genética no cromossomo 21 , que é causada quando a divisão celular acontece de forma anormal, resultando assim em um material extra no cromossomo 21. Normalmente, uma pessoa sem alteração genética tem 46 cromossomos em suas células e esses são recebidos pelas células embrionárias dos pais, no momento da fecundação. Assim, 


\section{QPUN}

23 vem dos espermatozoides e 23 vem dos óvulos, formando o zigoto/ovo, que se dividem e começam a formar um novo organismo (SILVA e DESSEN, 20020)

Existem 3 tipos de Síndrome de Down, nos quais são denominados: Trissomia 21, Mosaicismo e Translocação. Como é uma condição genética, as características começam a aparecer já na formação fetal. Na trissomia 21, todas as células vão apresentar um cromossomo extra no par de cromossomo 21. Já no mosaicismo, apenas uma parte das células vão apresentar o terceiro cromossomo 21. No tipo de SD por translocação, o par de cromossomo 21 vai continuar com apenas 2, mas uma parte do que seria o terceiro cromossomo, vai estar grudado, normalmente, no par de cromossomos 14 ou 22 (RAMOS, et al 2013).

1. Cariótipo de uma pessoa com Síndrome de Down do tipo Trissomia 21 e tipo Mosaicismo

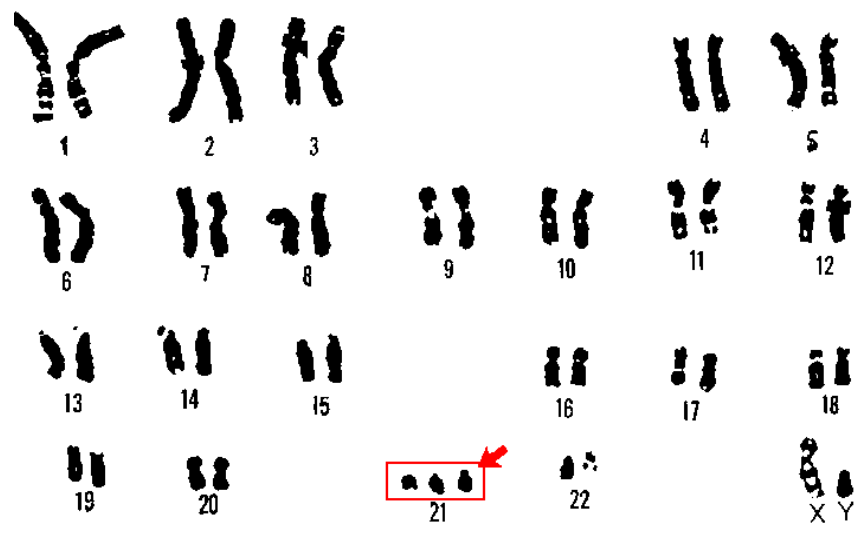

Referência: Google Imagens

2. Cariótipo de uma pessoa com Síndrome de Down do tipo Translocação 


\section{POFN}

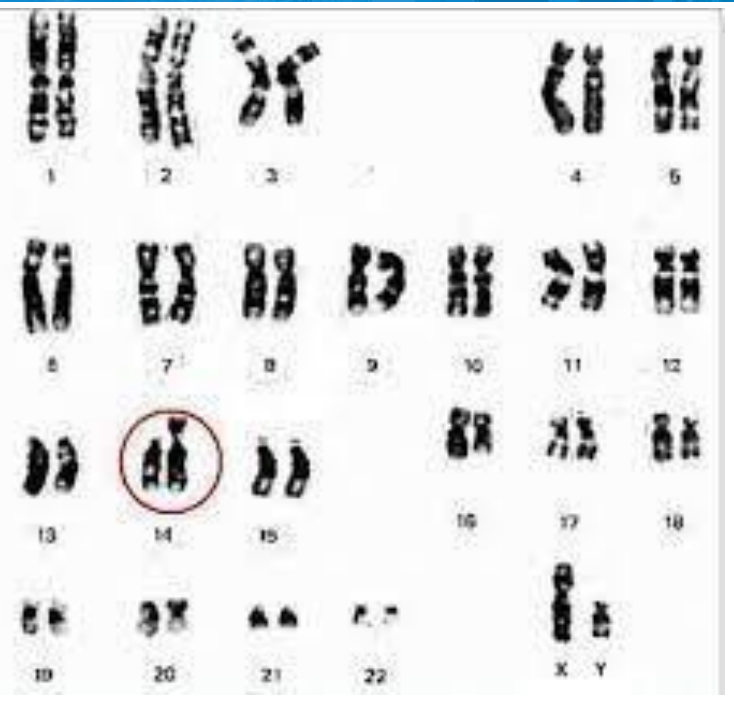

Referência: Google Imagens

Como podemos ver nas imagens acima, o cariótipo de uma pessoa com SD nos tipos de Trissomia 21 e Mosaicismo são iguais, a mudança vai ocorrer apenas na contagem das células com o cromossomo extra. Já no cariótipo da SD por Translocação, percebemos que o par de cromossomos 14 está com um pedaço a mais, que seria o terceiro cromossomo 21.

A Síndrome de Down é caracterizada por uma deficiência cognitiva, com traços e características físicas e psicológicas. Alguns exemplos são: olhos amendoados, um perfil reto, pescoço curto, orelhas com implantação mais baixa, mãos com linha única, hipotonia muscular, estatura mais baixa, deficiência intelectual e com maior tendência à obesidade. Mesmo que o tipo de SD seja diferente, as características são as mesmas. É sempre válido lembrar que a Síndrome de Down não é uma doença, mas sim, uma característica genética, na qual não tem cura. Para que o crescimento e desenvolvimento dessa criança aconteça da melhor forma possível, existem profissionais que podem ajudar e auxiliar no crescimento e desenvolvimento dessa criança (ASTEGIANO et al, 2019).

\section{Hipotonia Muscular}


A Vida com Logan

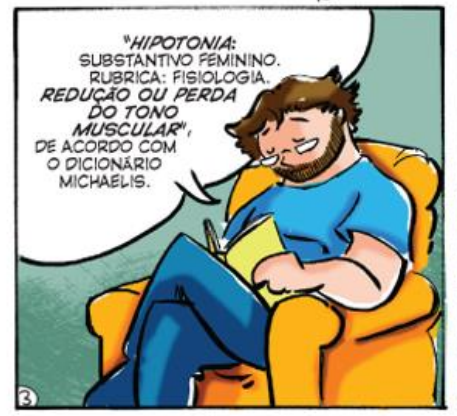

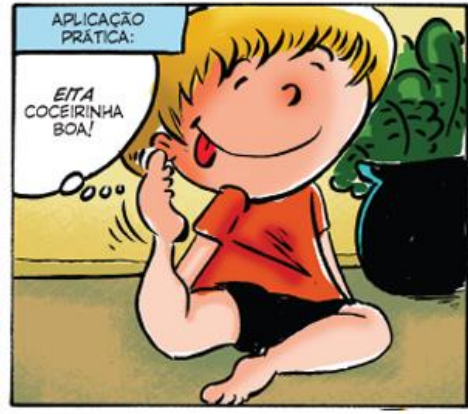

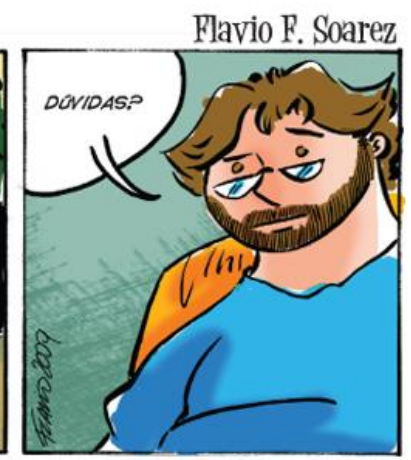

Fonte: Flávio Soares, 2016

A Enfermagem leva como essência da sua profissão o cuidado. Desse modo, para que a inclusão de pessoas com deficiência aconteça de forma harmônica e integral, é necessário que a equipe de Enfermagem esteja sempre adaptada, para entender as necessidades que podem ocorrer nessas situações (PAGLIUCA e MAIA, 2012).

Atender uma pessoa com SD, por exemplo, não é apenas cuidar do seu problema físico, é dar atenção, conversar e acalmar, pois existe um carinho e um amor muito grande vindo dela. É sempre muito importante desenvolver a Educação e Promoção da saúde, explicando os procedimentos, conversando com o paciente e seu familiar. Além de promover a comunicação terapêutica e acalmar o usuário, o profissional irá aprender muito, de modo, dando exemplo positivo para os outros profissionais da sua equipe (ALVES; PIRES; SERVO, 2013).

Como uma das características da SD é o atraso no desenvolvimento cognitivo e motor, é de extrema importância iniciar cedo os vários tipos de terapias, como a Terapia Ocupacional, Fonoaudiologia e Fisioterapia, pois são esses profissionais que irão ajudar tanto no crescimento e desenvolvimento da criança, quanto no apoio e aceitação da sua família. Esses profissionais além de ajudar a família, vão auxiliar na identificação de quais outras profissões são importantes para esse infante, assim trabalhando de uma maneira multiprofissional (HALBERSTADT; MORAES; SOUZA, 2020).

De acordo com Cunha, Blascovi-assis e Fiamenghi JR (2010), a chegada de uma criança com Síndrome de Down em uma família, pode causar um misto de 
sentimentos e emoções no qual, se percebe a necessidade do profissional da enfermagem, em desenvolver a escuta sensível promovendo acolhimento ao amenizar possíveis dúvidas e anseios.

O diálogo de saberes, dos profissionais de saúde com a família, deverá ser terapêutico, pois poderá emergir os primeiros sentimentos e incertezas, que devem ser percebidos de maneira verbal e não verbal, para que a afetividade amorosa possa ser vivenciada da melhor maneira possível. Cabe salientar que o exercício da interdisciplinaridade é de extrema importância, pois a sua efetividade contribui para o crescimento e desenvolvimento da criança com Síndrome de Down em harmonia, conforto e bem-estar com sua família (CUNHA; BLASCOVI-ASSIS; FIAMENGHI JR, 2010).

\section{CONCLUSÃO}

Ao socializar a construção do portfólio acadêmico no desenvolvimento da temática que atende as necessidades do cuidado com as pessoas com Síndrome de Down, entende-se a sua relevância no sentido de possibilitar ao estudante o efetivo sentido de refletir acerca das temáticas que emergem da formação profissional e que necessitam ser discutidas com protagonismo e autonomia.

\section{REFERÊNCIAS}

ASTEGIANO, C.; BOIARDI, A.; CACIOLI, J. P.; PIRERA, M., A., RAFAGHELLI, N., R., AGUSTINA, T., ALEJANDRO, T. Trisomía del par XXI: características estomatognáticas. Rev. Soc. Odontol. La Plata, 2019; Disponível em: <https://docs.bvsalud.org/biblioref/2020/02/1049947/revista solp 57 astegiano.pdf> Acesso em: 20 de agosto de 2021

ALVES, L., J., T. PIRES, A., N., M. SERVO, S., L., M. Um Olhar Sobre a Atuação do Enfermeiro na Atenção às Pessoas com Deficiência: Revisão Integrativa. Rev Enferm UFPE on line., Recife, 7(esp):4892-8, jul., 2013. Disponível em: <https://periodicos.ufpe.br/revistas/revistaenfermagem/article/view/11748/14020> Acesso em: 30 de julho de 2021 
CUNHA, V., F., M., A., BLASCOVI- ASSIS., M., S., FIAMENGHI JR, A., G. Impacto da notícia da síndrome de Down para os pais: histórias de vida. Ciênc. saúde coletiva, Rio de Janeiro , v. 15, n. 2, p. 445-451, Mar. 2010. Disponível em: $<$ http://www.scielo.br/scielo.php?script=sci arttext\&pid=S1413-81232010000200021 \&lng=en\&nrm=iso > Acesso em: 31 de julho de 2021

HANNUM, S., S., J. MIRANDA, J., F., SALVADOR, F., I., CRUZ, D., A. Impacto do diagnóstico nas famílias de pessoas com Síndrome de Down: revisão da literatura. Pensando fam., Porto Alegre, v. 22, n. 2, p. 121-136, dez. 2018. Disponível em: <http://pepsic.bvsalud.org/scielo.php?script=sci arttext\&pid=S1679494X2018000200009\&Ing=pt\&nrm=iso > Acesso em: 01 de agosto de 2021

HAlBeRstadT, F., B., MORAES, B., A., SOUZA., R., P., A. Síndrome De Down: Funcionalidade E Histórico Terapêutico Da Criança, Adaptação E Desempenho Ocupacional, Junho de 2020. Disponível em: <https://docs.bvsalud.org/biblioref/2021/03/1150530/7662-outros-50491-1-1020201120.pdf> Acesso em: 01 de agosto de 2021

LEITE, C., J. NEVES, J.,C.,J. VITOR, V.,G., L. FUJISAWA, S.,D. Controle postural em crianças com síndrome de down: avaliação do equilíbrio e da mobilidade funcional Rev. Bras. Ed. Esp., Marília, v. 24, n. 2, p.173-182, junho de 2018. Disponível em: <https://www.scielo.br/j/rbee/a/PrCJCGsnmrwgh85Qg7YHJkc/?lang=pt> Acesso em: 10 de setembro de 2021

MATIAS DELA CRUZ, M., L., SALVADOR, C., F., J., VELENZUELA, M., C., J., Importância da participação dos pais no treinamento de comunicação gestual para o desenvolvimento de habilidades de comunicação em crianças com síndrome de Down. Rev. chil. pediatr., Santiago, v. 91, n. 5 p. 840-842, outubro de 2020.

Disponível

em

$<$ https://scielo.conicyt.cl/scielo.php?script=sci arttext\&pid=S0370-

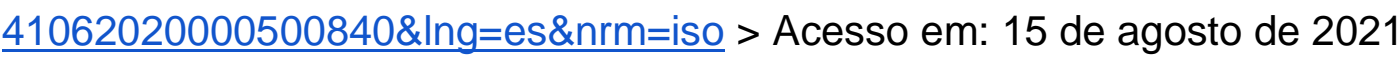


NUNES, R., D., M., DUPAS, G. Independência de crianças com síndrome de Down: as experiências de famílias. Rev. Latino-Am. Enfermagem, Ribeirão Preto, v. 19, n. 4, pág. 985-993, agosto de 2011. Disponível em $<$ http://www.scielo.br/scielo.php?script=sci arttext\&pid=S0104-

11692011000400018\&lng=en\&nrm=iso $>$. Acesso em: 30 de agosto de 2021

PAGLIUCA, F., M., L.; MAIA, R., E. Competência para prestar cuidado de enfermagem transcultural à pessoa com deficiência: instrumento de autoavaliação.

Disponível em:

<https://www.scielo.br/j/reben/a/cDkRTCgfkhZfZ689RsNg6gt/?lang=pt\&format=pdf> Acesso em: 10 de setembro de 2021

RAMOS, S., B. BONOMO, A., C. GOULART, A., M. ALMEIDA, P. “As DIFERENTES formas da trissomia 21." Movimento Down, 2013, Disponível em: $<$ http://www.movimentodown.org.br/movimento-down/equipe/> . Acesso em: $20 \mathrm{de}$ agosto de 2021

SANTANA, X., N., CAVALCANTE, J., Conceito neuroevolutivo em pacientes com síndrome de down: revisão integrativa. SALUSVITA, Bauru, v. 37, n. 4, p. 10091018 2018.

Disponível em:

https://secure.unisagrado.edu.br/static/biblioteca/salusvita/salusvita v37 n4 2018/sa lusvita v37 n4 2018 art 15.pdf > Acesso em: 03 de setembro de 2021

SILVA, N., L., P; DESSEN, M. A. Síndrome de Down: etiologia, caracterização e impacto na família. Curitiba, dez. 2002. ISSN 1981-8076. Disponível em: $<$ https://revistas.ufpr.br/psicologia/article/view/3304/2648> Acesso em: 10 de setembro de 2021 